\title{
LA POBLACION DE LOS MUNICIPIOS ESPAÑOLES
}

352: 312

por

Ignacio Ballester Ros

SUMARIO: I. INTRODUCCION.-II. LA DISTRIBUCION DE LOS MUNICIPIOS Y DE SU POBLACION EN EL CONJUNTO NACIONAL.-III. LA DISTRIBUCION DE LOS MUNICIPIOS EN LAS PROVINCIAS Y EN LAS COMUNIDADES AUTONOMAS.IV. LA DISTRIBUCION DE LA POBLACION DE LOS MUNICIPIOS EN LAS PROVINCIAS Y EN LAS COMUNIDADES AUTONOMAS.

\section{INTRODUCCION}

En el número 213 de esta Revista se publicó un trabajo sobre la Evolución de la Población Española en el periodo 1971-1981, tomando como base de información las cifras oficiales de población, resultantes del Censo de Población realizado por el Instituto Nacional de Estadística con referencia al 1 de marzo de 1981, y declaradas oficiales por el Real Decreto 3114/1981, de 27 de noviembre.

Se anunciaba ya que el Instituto Nacional de Estadística estaba preparando la monografía en que se harian públicas las poblaciones oficiales de derecho y de hecho de cada uno de los Municipios españoles, monografía que se ha editado bajo el título de "Poblaciones de derecho y de hecho de los Municipios españoles. Censo de 
Población de 1981"; y al propio tiempo se han publicado algunas tablas elaboradas sobre dichos datos en la monografía del propio Instituto Nacional de Estadística titulada «España. Anuario Estadístico 1982».

La información estadística contenida en las dos monografias reseñadas nos permite abordar esta crónica, dedicada al análisis de la distribución de la población española en el área municipal, según el tamaño demográfico de los Municipios y su ubicación en las Provincias y en las Comunidades Autónomas.

De esta forma, podremos fijar algunas conclusiones sobre el asentamiento de la población, que constituye uno de los elementos básicos del Municipo, sobre el área territorial.

\section{LA DISTRIBUCION DE LOS MUNICIPIOS Y DE SU POBLACION EN EL CONJUNTO NACIONAL}

Según el Censo de la Población de España de 1981, el número de Municipios existentes en el momento censal era de

\subsection{2}

y la población censada, de hecho, fue de

\subsection{6 .260}

habitantes.

Tomando como base la información contenida en el expresado "Anuario Estadístico de España», hemos elaborado la siguiente tabla:

\begin{tabular}{|c|c|c|c|c|c|}
\hline & TAMANO DE LOS MUNICIPIOS & $\begin{array}{c}\text { Número } \\
\text { de } \\
\text { Municipios }\end{array}$ & $\begin{array}{l}\text { Porcen- } \\
\text { tajes }\end{array}$ & $\begin{array}{l}\text { Población } \\
\text { de los } \\
\text { Municipios }\end{array}$ & $\begin{array}{c}\text { Potcen } \\
\text { tajes }\end{array}$ \\
\hline $\begin{array}{l}\text { De } \\
\text { De } \\
\text { De } \\
\text { De } \\
\text { De } \\
\text { De } \\
\text { De } \\
\text { De } \\
\mathrm{De} \\
\mathrm{De} \\
\mathrm{De} \\
\mathrm{De} \\
\mathrm{De}\end{array}$ & 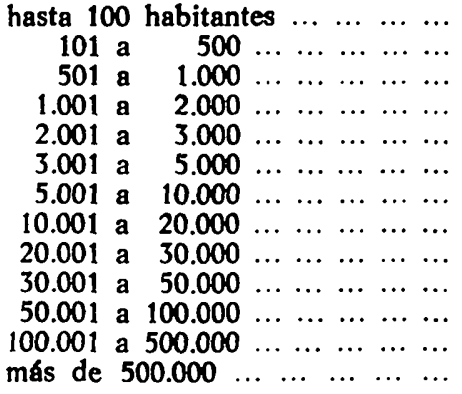 & $\begin{array}{r}682 \\
2.849 \\
1.303 \\
1.059 \\
559 \\
517 \\
513 \\
288 \\
97 \\
52 \\
53 \\
44 \\
6\end{array}$ & $\begin{array}{r}8,5 \\
35,5 \\
16,3 \\
13,2 \\
7,0 \\
6,4 \\
6,4 \\
3,6 \\
1,2 \\
0,6 \\
0,7 \\
0,5 \\
0,1\end{array}$ & $\begin{array}{r}42.684 \\
756.666 \\
932.867 \\
1.513 .792 \\
1.360 .693 \\
1.983 .929 \\
3.524 .103 \\
3.954 .716 \\
2.325 .237 \\
1.966 .832 \\
3.521 .466 \\
8.420 .510 \\
7.442 .765\end{array}$ & $\begin{array}{r}0.1 \\
2,0 \\
2,5 \\
4,0 \\
3,6 \\
5,3 \\
9,3 \\
10,5 \\
6,2 \\
5,2 \\
9,3 \\
22,3 \\
19,7\end{array}$ \\
\hline
\end{tabular}


Si reducimos esta tabla, utilizando niveles de población más acordes y de mayor significación desde el punto de vista de la legislación de Régimen local, obtenemos ésta:

\begin{tabular}{|c|c|c|c|c|c|c|}
\hline TAMANO DE LOS MUNICIPIOS & $\begin{array}{c}\text { Número } \\
\text { de } \\
\text { Municipios }\end{array}$ & Porcer & taies & $\begin{array}{l}\text { Población } \\
\text { de los } \\
\text { Municipios }\end{array}$ & Porcen & taies \\
\hline 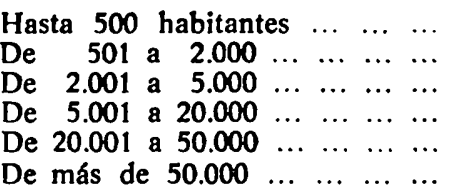 & $\begin{array}{r}3.531 \\
2.362 \\
1.076 \\
801 \\
149 \\
103\end{array}$ & $\begin{array}{r}\left.\begin{array}{r}44,0 \\
29,5 \\
13,4 \\
10,0 \\
1,8\end{array}\right\}\end{array}$ & $\begin{array}{r}86,9 \\
11,8 \\
1,3\end{array}$ & $\begin{array}{r}799.350 \\
2.446 .659 \\
3.344 .422 \\
7.478 .819 \\
4.292 .069 \\
19.384 .741\end{array}$ & $\left.\begin{array}{r}2,1 \\
6,5 \\
8,9 \\
19,8 \\
11,4\end{array}\right\}$ & 17,5 \\
\hline
\end{tabular}

Del examen de las tablas anteriores resalta, en primer término, la acusada falta de homogeneidad de los Municipios españoles, en orden a su tamaño demográfico. Coexisten Municipios minúsculos juntamente con grandes ciudades y lógicamente resulta quimérica la igualdad de todos los españoles en orden a la disponibilidad de disfrute de los servicios públicos comunitarios, que no puede darse, en el mismo grado, para toda la población española.

En segundo lugar, se advierte que la distribución de los Municipios, según su tamaño, y de sus respectivas poblaciones, con arreglo a los niveles o estratos considerados, están en razón inversa.

Mientras que los Municipios se concentran, en cuanto a su número, de manera predominante, en los estratos más bajos en cuanto a la cifra de sus habitantes, la población de estos Municipios se concentra, por el contrario, en un número relativamente reducido de Municipios de tamaño medio y, muy especialmente, de grandes urbes.

Pudiéramos afirmar, en tercer término, que de cada 100 Municipios, 87 tienen una población inferior a los 5.000 habitantes; 12 oscilan entre 5.001 y 50.000 habitantes; y uno supera los 50.000 .

En cuarto lugar, conviene destacar que más de la mitad de la población reside en Municipios mayores de $\mathbf{5 0 . 0 0 0}$ habitantes; casi una tercera parte se ubica en Municipios de tamaño medio, entre 5.001 y 50.000 habitantes; y algo más de la sexta parte habita en Municipios de hasta 5.000 habitantes. 


\section{LA DISTRIBUCION DE LOS MUNICIPIOS EN LAS PROVINCIAS Y EN LAS COMUNIDADES AUTONOMAS}

La información disponible permite elaborar una distribución de los Municipios con arreglo a los estratos considerados en el apartado anterior. Sin embargo, hemos juzgado preferible en este trabajo, de extensión limitada, reducir las tablas a los tres grandes grupos de Municipios de mayor significación.

A estos efectos, hemos elaborado la siguiente tabla provincial:

\begin{tabular}{|c|c|c|c|c|}
\hline \multirow[b]{2}{*}{ PROVINCIAS } & \multirow[b]{2}{*}{ Total } & \multicolumn{3}{|c|}{ NUMERO DE MUNICIPIOS } \\
\hline & & $\begin{array}{c}\text { De hasta } \\
5.000 \\
\text { habitantes }\end{array}$ & $\begin{array}{c}\text { De } 5.001 \\
\text { a } 50.000 \\
\text { habitantes }\end{array}$ & $\begin{array}{c}\text { Mayores } \\
\text { de } 50.000 \\
\text { habitantes }\end{array}$ \\
\hline & & Cifras & osolutas & \\
\hline $\begin{array}{llllllllll}\text { Alava } & \ldots & \ldots & \ldots & \ldots & \ldots & \ldots & \ldots & \ldots\end{array}$ & 51 & 48 & 2 & 1 \\
\hline Albacete $\ldots \ldots \ldots \ldots \ldots \ldots$ & 86 & 77 & 8 & 1 \\
\hline 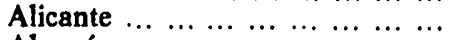 & 138 & 95 & 39 & 4 \\
\hline 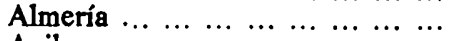 & 102 & 90 & 11 & 1 \\
\hline $\begin{array}{llllllllllll}\text { Avila } & \ldots & \ldots & \ldots & \ldots & \ldots & \ldots & \ldots & \ldots & \ldots\end{array}$ & 247 & 243 & 4 & - \\
\hline $\begin{array}{lllllllll}\text { Badajoz } & \ldots & \ldots & \ldots & \ldots & \ldots & \ldots & \ldots\end{array}$ & 162 & 134 & 27 & 1 \\
\hline $\begin{array}{llllllllllllllllll}\text { Baleares } & \ldots & \ldots & \ldots & \ldots & \ldots & \ldots & \ldots & \ldots\end{array}$ & 65 & 38 & 26 & $i$ \\
\hline $\begin{array}{llllllll}\text { Barcelona } & \ldots & \ldots & \ldots & \ldots & \ldots & \ldots & \ldots\end{array}$ & 307 & 224 & 71 & 12 \\
\hline $\begin{array}{llllllllll}\text { Burgos } & \ldots & \ldots & \ldots & \ldots & \ldots & \ldots & \ldots & \ldots\end{array}$ & 374 & 369 & 4 & 1 \\
\hline $\begin{array}{lllllllll}\text { Cáceres } & \ldots & \ldots & \ldots & \ldots & \ldots & \ldots & \ldots & \ldots\end{array}$ & 218 & 206 & 11 & 1 \\
\hline $\begin{array}{llllllllll}\text { Cádiz } & \ldots & \ldots & \ldots & \ldots & \ldots & \ldots & \ldots & \ldots\end{array}$ & 42 & 13 & 23 & 6 \\
\hline $\begin{array}{llllllll}\text { Castellón } & \ldots & \ldots & \ldots & \ldots & \ldots & \ldots & \ldots\end{array}$ & 134 & 122 & 11 & 1 \\
\hline Ciudad Real $\ldots \begin{array}{lllll} & \ldots & \ldots & \ldots & \ldots\end{array}$ & 98 & 71 & 26 & 1 \\
\hline $\begin{array}{lllllllll}\text { Córdoba } & \ldots & \ldots & \ldots & \ldots & \ldots & \ldots & \ldots\end{array}$ & 75 & 46 & 28 & 1 \\
\hline $\begin{array}{ccccccc}\text { Coruña, La } & \ldots & \ldots & \ldots & \ldots & \ldots & \ldots\end{array}$ & 93 & 32 & 58 & 3 \\
\hline $\begin{array}{llllllll}\text { Cuenca } & \ldots & \ldots & \ldots & \ldots & \ldots & \ldots & \ldots\end{array}$ & 234 & 228 & 6 & - \\
\hline $\begin{array}{lllllllll}\text { Gerona } & \ldots & \ldots & \ldots & \ldots & \ldots & \ldots & \ldots & \cdots\end{array}$ & 219 & 201 & 17 & 1 \\
\hline 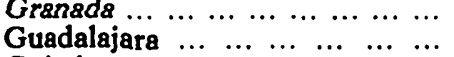 & $\begin{array}{l}166 \\
289\end{array}$ & $\begin{array}{l}140 \\
286\end{array}$ & $\begin{array}{r}25 \\
2\end{array}$ & 1 \\
\hline $\begin{array}{lllllll}\text { Guipúzcoa } & \ldots & \ldots & \ldots & \ldots & \ldots & \ldots\end{array}$ & 81 & 50 & 29 & 2 \\
\hline $\begin{array}{lllllllll}\text { Huelva } & \ldots & \ldots & \ldots & \ldots & \ldots & \ldots & \ldots & \ldots\end{array}$ & 79 & 59 & 19 & 1 \\
\hline $\begin{array}{ccccccccc}\text { Huesca } & \ldots & \ldots & \ldots & \ldots & \ldots & \ldots & \ldots & \ldots\end{array}$ & 200 & 193 & 7 & - \\
\hline $\begin{array}{llllllllll} & \operatorname{Jaén} & \ldots & \ldots & \ldots & \ldots & \ldots & \ldots & \ldots & \ldots\end{array}$ & 96 & 63 & 31 & 2 \\
\hline $\begin{array}{llllllllll}\text { Lebn } & \ldots & \ldots & \ldots & \ldots & \ldots & \ldots & \ldots & \ldots & \ldots\end{array}$ & 213 & 201 & 10 & 2 \\
\hline $\begin{array}{llllllllll}\text { Lérida } & \ldots & \ldots & \ldots & \ldots & \ldots & \ldots & \ldots & \ldots\end{array}$ & 229 & 219 & 9 & 1 \\
\hline $\begin{array}{lllllllll}\text { Lugo } & \ldots & \ldots & \ldots & \ldots & \ldots & \ldots & \ldots & \ldots\end{array}$ & 66 & 43 & 22 & 1 \\
\hline $\begin{array}{lllllllll}\text { Madrid } & \ldots & \ldots & \ldots & \ldots & \ldots & \ldots & \ldots & \ldots\end{array}$ & 178 & 144 & 23 & 11 \\
\hline $\begin{array}{llllllllll}\text { Málaga } & \ldots & \ldots & \ldots & \ldots & \ldots & \ldots & \ldots & \ldots\end{array}$ & 99 & 79 & 18 & 2 \\
\hline $\begin{array}{llllllllll}\text { Murcia } & \ldots & \ldots & \ldots & \ldots & \ldots & \ldots & \ldots & \ldots\end{array}$ & 44 & 10 & 31 & 3 \\
\hline $\begin{array}{llllllllll}\text { Navarra } & \ldots & \ldots & \ldots & \ldots & \ldots & \ldots & \ldots & \ldots\end{array}$ & 264 & 252 & 11 & 1 \\
\hline $\begin{array}{llllllllll}\text { Orense } & \ldots & \ldots & \ldots & \ldots & \ldots & \ldots & \ldots & \ldots\end{array}$ & 92 & 73 & 18 & 1 \\
\hline $\begin{array}{llllllll}\text { Oviedo } & \ldots & \ldots & \ldots & \ldots & \ldots & \ldots & \ldots\end{array}$ & 78 & 43 & 30 & 5 \\
\hline 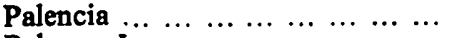 & 191 & 187 & 3 & 1 \\
\hline $\begin{array}{lllllll}\text { Palmas, Las } & \ldots & \ldots & \ldots & \ldots & \ldots & \ldots\end{array}$ & 34 & 12 & 19 & 3 \\
\hline $\begin{array}{llllllll}\text { ontevedra } & \ldots & \ldots & \ldots & \ldots & \ldots & \ldots & \ldots\end{array}$ & 61 & 18 & 41 & 2 \\
\hline $\begin{array}{lllllllll}\text { Rioja } & \ldots & \ldots & \ldots & \ldots & \ldots & \ldots & \ldots & \ldots\end{array}$ & 174 & 167 & 6 & 1 \\
\hline $\begin{array}{llllllll}\text { Salamanca } & \ldots & \ldots & \ldots & \ldots & \ldots & \ldots & \ldots\end{array}$ & 357 & 353 & 3 & 1 \\
\hline
\end{tabular}




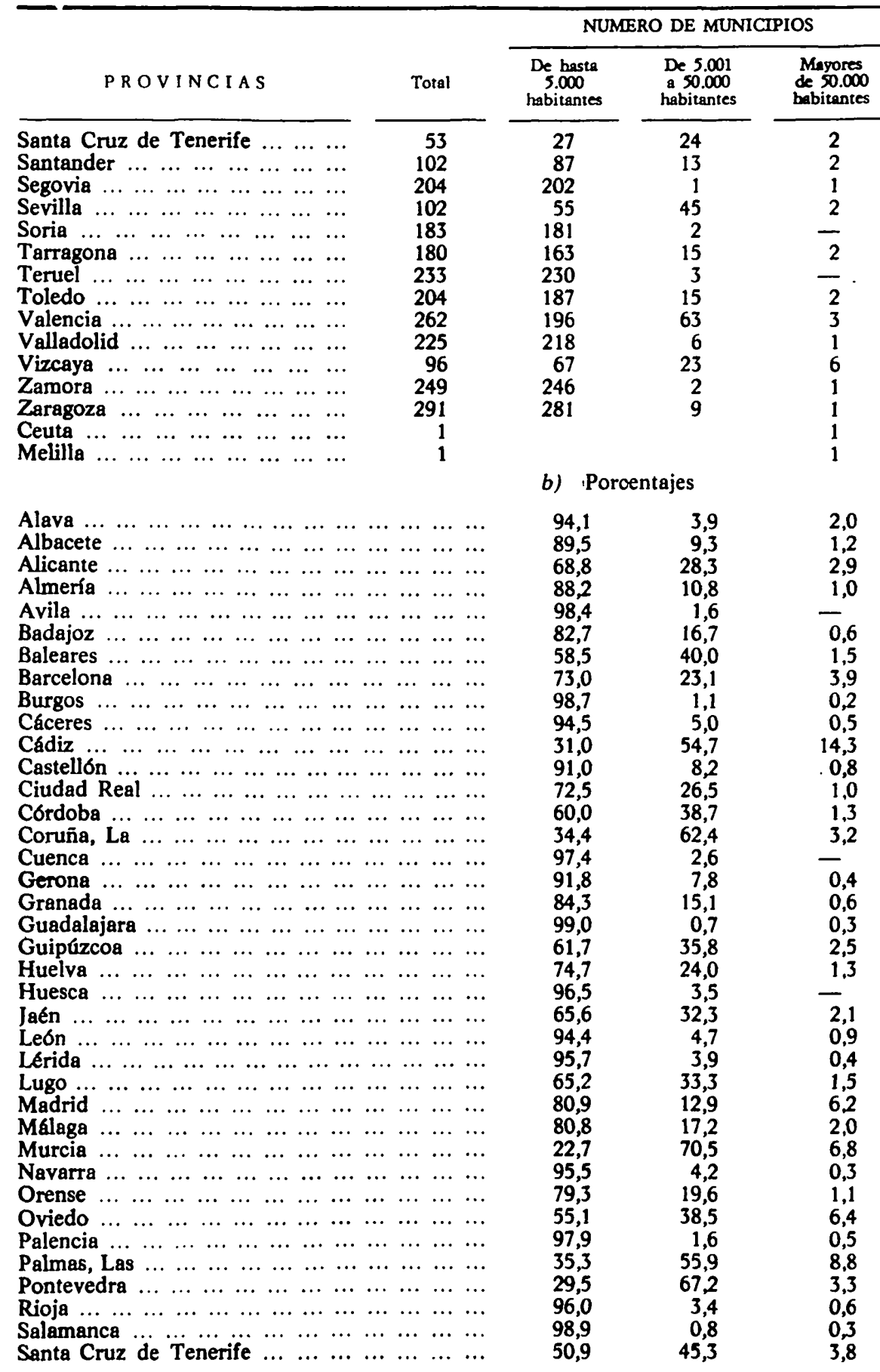




\begin{tabular}{|c|c|c|c|}
\hline \multirow[b]{2}{*}{ PROVINCIAS } & \multicolumn{3}{|c|}{ NUMERO DE MUNICIPIOS } \\
\hline & $\begin{array}{l}\text { De hasta } \\
5.000 \\
\text { habitantes }\end{array}$ & $\begin{array}{c}\text { De } 5.001 \\
\text { a } 50.000 \\
\text { habitantes }\end{array}$ & $\begin{array}{l}\text { Mayores } \\
\text { de } \$ 0.000 \\
\text { habitantes }\end{array}$ \\
\hline 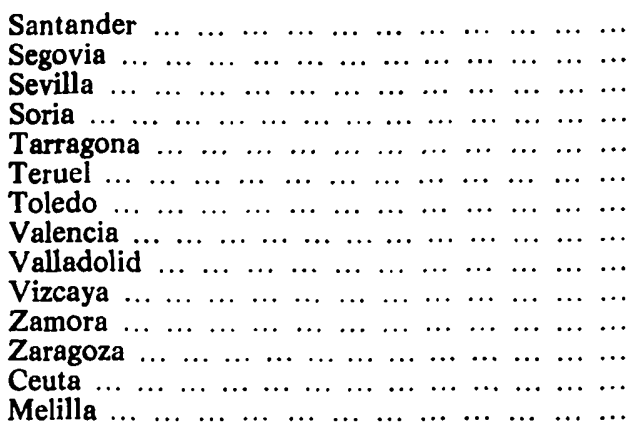 & $\begin{array}{l}85,3 \\
99,0 \\
53,9 \\
98,9 \\
90,6 \\
98,7 \\
91,7 \\
74,8 \\
96,9 \\
69,8 \\
98,8 \\
96,6\end{array}$ & $\begin{array}{r}12,7 \\
0,5 \\
44,1 \\
1,1 \\
8,3 \\
1,3 \\
7,4 \\
24,1 \\
2,7 \\
24,0 \\
0,8 \\
3,1\end{array}$ & $\begin{array}{r}2,0 \\
0,5 \\
2,0 \\
-1,1 \\
-0,9 \\
1,1 \\
0,4 \\
6,2 \\
0,4 \\
0,3 \\
100,0 \\
100,0\end{array}$ \\
\hline
\end{tabular}

Se observa, en primer término, que el área territorial en la que predominan los pequeños Municipios de hasta 5.000 habitantes, comprende, en principio, a 25 Provincias, que geográficamente se distribuyen en esta forma:

- La Submeseta Norte, en toda su integridad.

- La Submeseta Sur, con la excepción de Madrid y Badajoz, que cuentan, sin embargo, con un número importante de Municipios de este nivel, aun cuando no superan el promedio nacional.

- El Valle del Ebro, íntegro.

- Litoral oriental mediterráneo: Gerona, Tarragona, Castellón y Almería.

Se trata de una distribución predominante central, que cubre dos grandes Regiones geográficas - la Meseta y el Valle del Ebro- y se prolonga hacia el Mediterráneo, constituyendo una muy extensa área geográfica, sin solución de continuidad.

Ello no quiere decir que no haya núcleos importantes de Municipios de este tamaño en otras Provincias, como Granada, Santander e incluso Madrid, entre otras.

Detallando algo más en el examen de este grupo de Municipios, se advierte que esta distribución coincide, en general, con la de predominio de los Municipios minúsculos de solo hasta 500 habitantes, algo más de 3.500 en toda España, con un promedio teórico de 71 por Provincia, que se ubican preferentemente en el Valle del Duero -excepto la Provincia de León-, Cuenca y Guadalajara en la Submeseta Sur, el Valle del Ebro — salvo Alava- y la Cataluña litoral. 
Los Municipios de población comprendida entre 501 y 2.000 habitantes, unos 2.350 , con un predominio teórico de 47 por Provincia, son más numerosos en el ámbito de la gran área descrita, en especial en las Provincias de León y Zamora, Toledo y Cáceres, y Valencia; y en segundo grado se sitúan las restantes Provincias del Valle del Duero, Madrid, Navarra con las de Aragón y Cataluña, Castellón, y aisladamente, Santander y Granada, estas últimas fuera de dicha área.

Los Municipios cuya población oscila entre 2.001 y 5.000 habitantes, algo más de 1.000 , con un promedio teórico de 21 por Provincia, se extienden ya, por el contrario, en un mayor número de Provincias, fuera, en parte, del área territorial básica a que nos hemos referido, propia del grupo de Municipios de hasta 5.000 habitantes y de los dos primeros subgrupos considerados y configuran estas zonas geográficas:

- Noroeste y Norte.-Galicia - menos Pontevedra-, Oviedo y Santander.

- La Submeseta Sur, salvo Ciudad Real.

- Litoral oriental mediterráneo.-Las Provincias litorales catalanas, Baleares y la Provincia de Valencia.

- Andalucía, excepto Cádiz.

- Santa Cruz de Tenerife.

Aisladamente se mantienen, además, León y Navarra.

Sobresalen, entre todas ellas, Oviedo, Barcelona y Valencia.

Los Municipios de tamaño medio, entre 5.001 y 50.000 habitantes, unos 950, con un promedio teórico de 19 por Provincia, predominan en 20 Provincias, cuya distribución geográfica se asemeja mucho a la del subgrupo que acabamos de describir, de los Municipios entre 2.001 y 5.000 habitantes, ya que configuran estas zonas:

- Noroeste y Norte.-Galicia y la orla cantábrica, en su integridad.

- Submeseta Sur.-Madrid, Badajoz y Ciudad Real.

- Litoral oriental mediterráneo.-Barcelona, Valencia, Alicante, Murcia y Baleares.

- Andalucía, en toda su integridad.

- Canarias.

Los valores más altos, por el peso de los Municipios de este tamaño en el total provincial, corresponden, ante todo, a Murcia, 
La Coruña y Pontevedra, las dos canarias, Cádiz y Sevilla, seguidas de Baleares, Oviedo, Córdoba y Jaén, Guipúzcoa y Lugo.

Cabe distinguir dentro de este grupo dos subgrupos. El primero, de los Municipios con población comprendida entre 5.001 y 20.000 habitantes, y el segundo, de los Municipios que tienen entre 20.001 y 50.000 .

En el primer subgrupo, que comprende 800 Municipios, con un promedio teórico de 16 por Provincia, sobresalen La Coruña, Pontevedra y Lugo; Barcelona, Valencia y Alicante; y Sevilla.

En el segundo subgrupo, que abarca 150 Municipios, con un promedio teórico de tres, destacan Barcelona, Valencia, Alicante y Murcia; y Sevilla y Cádiz.

Los Municipios de población superior a 50.000 habitantes se ubican preferentemente en 20 Provincias, que geográficamente configuran estas zonas:

- Noroeste y orla cantábrica.-En su integridad, salvo Orense.

- Madrid.

- Litoral oriental mediterráneo.-Barcelona, Alicante, Murcia y Baleares.

- Andalucía, salvo Almería, Granada y Córdoba.

- Canarias.

Como se observa, estos Municipios tienen una distribución territorial similar en líneas generales a los del grupo anterior.

Sobresalen por la importancia de estos Municipios en el conjunto provincial las Provincias de Oviedo y Vizcaya, Madrid, Murcia y Cádiz.

Cabe, a su vez, distinguir en este grupo tres subgrupos. El primero comprende los Municipios de 50.001 a 100.000 habitantes; el segundo los de 100.001 a 500.000 , y el tercero los superiores a medio millón.

Los Municipios del primer subgrupo, 53, se hallan situados solamente en 28 Provincias, entre las que sobresalen Madrid y Barcelona, Vizcaya y Cádiz.

Los Municipios del segundo subgrupo, 44, se sitúan en 30 Provincias, entre las que destacan Oviedo y Vizcaya; Madrid; Barcelona, Alicante y Murcia; Cádiz y Santa Cruz de Tenerife.

Los Municipios del tercer subgrupo son solamente seis: Madrid y Barcelona; Valencia y Zaragoza; Sevilla y Málaga, que constituyen las grandes urbes españolas. 
Para concretar mejor la configuración de las grandes áreas territoriales afectadas por la distribución de los Municipios de los tres grandes grupos considerados, hemos elaborado la siguiente tabla, referente a las Comunidades Autónoma:

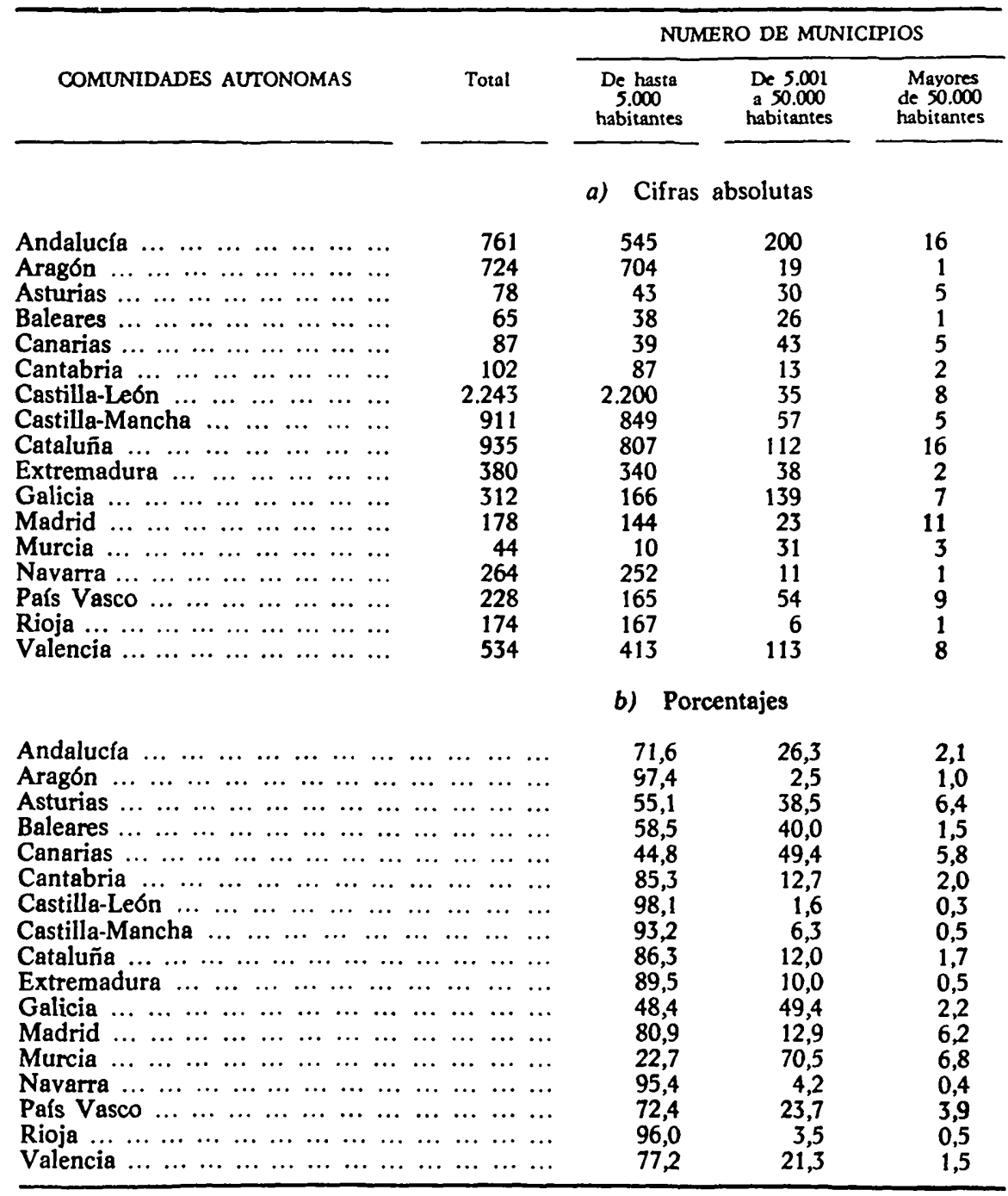

Observamos que con esta tabla queda ratificada la distribución de los Municipios de hasta 5.000 habitantes, que predominan, con valores superiores al promedio nacional -86,9-, en las siguientes Regiones: 
- La Meseta, comprendiendo las Comunidades Autónomas de Castilla-León, Castilla-Mancha y Extremadura.

- El Valle del Ebro, abarcando las Comunidades Autónomas de Navarra, Rioja y Aragón.

Consignemos como complemento de lo expuesto que las Comunidades Autónomas de Cantabria, Cataluña y Madrid se acercan al promedio nacional, sin rebasarlo, como expresión de la disponibilidad de un fuerte contingente de Municipios de este nivel.

Los Municipios de población comprendida entre los 5.001 y 50.000 habitantes se distribuyen también ratificando lo expuesto al analizar las Provincias, y configuran las siguientes áreas:

- Noroeste y Norte.-Galicia y Asturias, Cantabria y el País Vasco, las dos primeras destacadas con valores muy altos.

- Litoral oriental mediterráneo.-Cataluña, Valencia, Murcia y Baleares, la penúltima con el valor más alto de la distribución, siguiéndole en importancia la última reseñada.

- Andalucía.

- Canarias, también muy destacada.

Los Municipios de población superior a los 50.000 habitantes se sitúan entre las Comunidades Autónomas de forma similar a la que acabamos de describir con respecto a los Municipios de tamaño medio, aunque añadiendo a Madrid, con un valor muy alto, sólo superado por Murcia, y seguida, muy de cerca, por Asturias, Canarias, el País Vasco y Galicia.

Se perfilan, pues, dos grandes áreas. La primera, interior, comprensiva de las dos grandes Regiones naturales de la Meseta y el Valle del Ebro, con predominio de los pequeños Municipios. La segunda, periférica, que comprende Galicia y la orla cantábrica, el litoral oriental mediterráneo, Andalucía y Canarias, a las que debe añadirse por excepción a Madrid.

\section{LA DISTRIBUCION DE LA POBLACION DE LOS MUNICIPIOS EN LAS PROVINCIAS Y EN LAS COMUNIDADES AUTONOMAS}

Examinada la distribución de los Municipios, según los estratos considerados y las áreas territoriales predominantes, parece mucho más interesante y significativo el análisis de la distribución de la población de esos mismos Municipios, igualmente encuadrados en los niveles contemplados. 
Para ello hemos elaborado la siguiente tabla provincial:

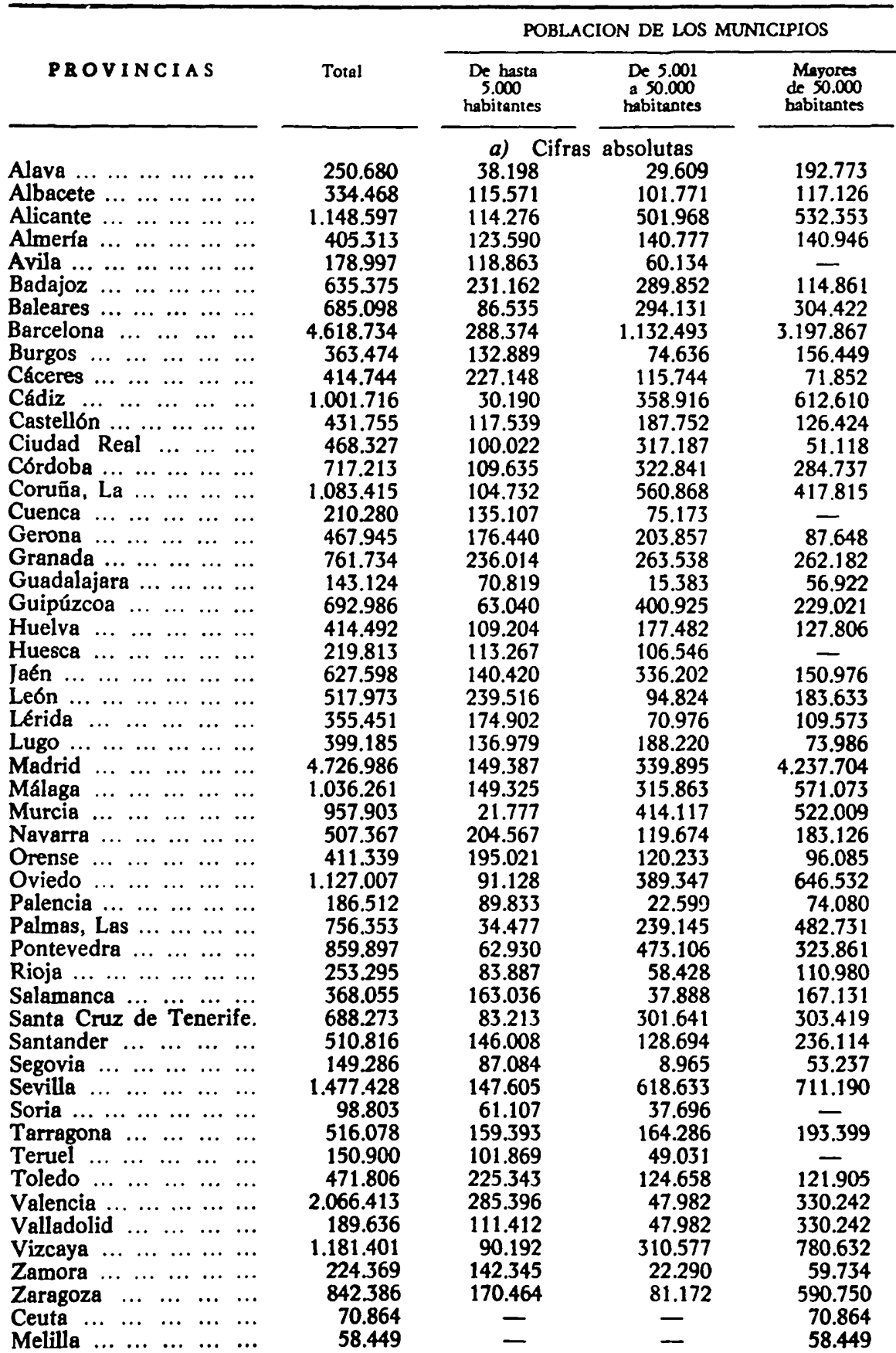




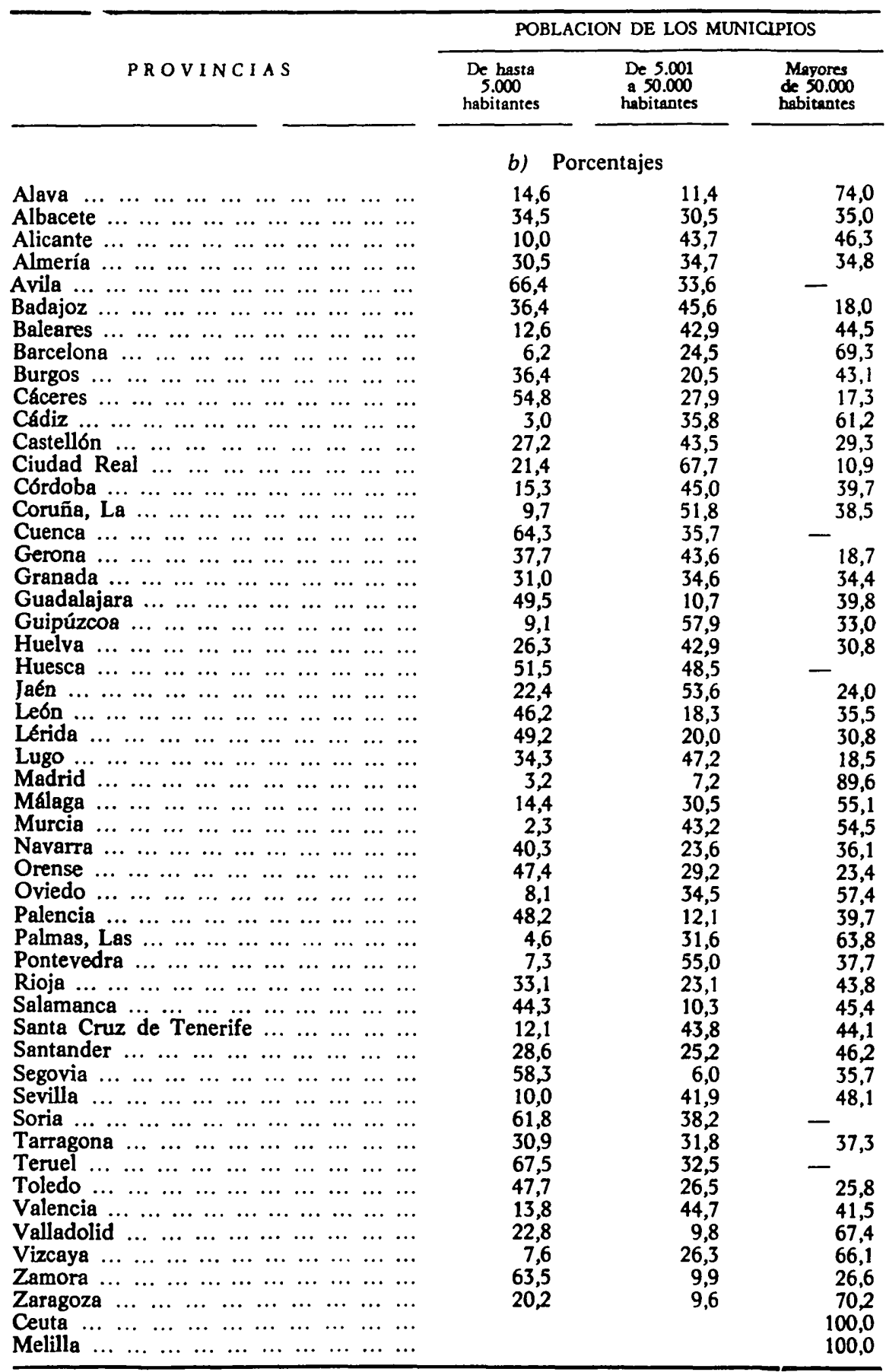


La observación de la tabla anterior nos permite afirmar que, en principio, la población residente en pequeños Municipios de hasta 5.000 habitantes es predominante en 32 Provincias, que geográficamente se distribuyen en forma básicamente similar a la de los Municipios del mismo grupo, aunque con algunas matizaciones. Configuran las siguientes zonas:

- Noroeste y Norte.-Lugo, Orense y Santander.

- Submeseta Norte, en toda su integridad.

- Submeseta Sur, con la excepción de Madrid.

- Valle del Ebro, salvo Alava. tellón.

- Litoral oriental mediterráneo--Gerona, Tarragona y Cas-

- Andalucía.-Almería, Granada, Jaén y Huelva.

La configuración geográfica, basada en el número de Municipios, se amplía, al considerar la población de dichos Municipios, a la Galicia interior y a Santander, en el norte, y a Andalucía oriental y parte de la occidental, al sur.

Alrededor de las dos terceras partes de la población de las Provincias de Zamora, Avila y Soria, en el Valle del Duero, y de Teruel y Cuenca residen en Municipios menores de 5.000 habitantes. Igualmente más de la mitad de la población de Segovia y de Cáceres reside en Municipios del mismo nivel. Más del 40 por 100 de la población de las Provincias de Orense; León, Palencia y Salamanca; Guadalajara y Toledo; Navarra y Lérida, habita en este tipo de Municipios. Y representa alrededor de la tercera parte de su población respectiva en ocho Provincias más.

Dentro del grupo de Municipios que contemplamos, la población respectiva reside preferentemente en Municipios de hasta 500 habitantes o de 501 a 2.000 en las Provincias de la Submeseta Norte y parte septentrional de la Submeseta Sur. Por el contrario, hay un predominio de residencia de esta población en Municipios de 2.001 a 5.000 habitantes en las Provincias de Lugo, Orense y León; Albacete, Ciudad Real y Toledo; Gerona, Tarragona y Castellón.

La población residente en Municipios de tipo medio, entre 5.001 y 50.000 habitantes, resulta predominante en 29 Provincias, distribuidas en esta forma:

- Noroeste y Norte.-La Coruña, Pontevedra y Lugo; Oviedo y Guipúzcoa. 
- Submeseta Norte.-Avila y Soria.

- Submeseta Sur.-Badajoz, Ciudad Real y Cuenca.

- Litoral oriental mediterráneo.-Gerona, Tarragona, Castellón, Valencia, Alicante, Murcia y Baleares.

- Andalucía, en su integridad.

- Canarias.

Comparando esta distribución con la anterior se observa que queda reducida el área en ambas Submesetas y en el Valle del Ebro, mientras predomina la Galicia marítima y se prolonga la orla cantábrica, y se extiende la Región litoral oriental mediterránea a su integridad, con la excepción de Barcelona; lo propio ocurre con Andalucía y aparecen las Canarias.

Residen en Municipios de este grupo las dos terceras partes de la población de Ciudad Real; la mitad de los habitantes de La Coruña, Pontevedra, Guipúzcoa y Jaén; más del 40 por 100 de la población de Gerona, Castellón, Valencia, Alicante, Murcia y Baleares; Badajoz; Córdoba, Sevilla y Huelva; y separadas entre sí, Lugo, Huesca y Santa Cruz de Tenerife; además de otras 12 Provincias, cuya tercera parte de la población habita en Municipios de este tamaño.

Dentro del grupo de Municipios que consideramos, la población residente en Municipios de 5.001 a 20.000 habitantes se acerca a los siete millones y medio, mientras que la ubicada en Municipios de 20.001 a 50.000 representa sólo 4,2 millones. Mientras que la población del primer subgrupo se extiende a todas las Provincias, la del segundo se limita a 35 Provincias, ya que 15 de ellas carecen de Municipios de este segundo subgrupo, en el que figuran, sin embargo, las capitales de Provincia, cuya población no alcanza los 50.000 habitantes.

La población residente en Municipios superiores a 50.000 habitantes afecta a 45 Provincias y predomina en 11: Oviedo, Alava, Vizcaya y Zaragoza; Madrid y Valladolid; Barcelona y Murcia; Cádiz. y Málaga; y Las Palmas. En todas ellas la población residente de este grupo supera el 50 por 100 de la población total de cada Provincia. En siete Provincias tal colectivo supera las dos terceras partes de la población provincial, y en el caso más significativo, Madrid, representa el 90 por 100 del total.

Cabe distinguir, dentro de este grupo, tres subgrupos. El primero corresponde a la población residente en Municipios de 50.001 a 100.000 habitantes $-3,5$ millones-, que afecta a 28 Provincias. 
El segundo, de población residente de 100.001 a 500.000 habitantes $-8,4$ millones-, que afecta solamente a 31 Provincias. Y el tercero, la población superior a medio millón $-7,4$ millones-, limitada a las seis urbes que superan esta cifra: Madrid, Barcelona, Valencia, Málaga, Sevilla y Zaragoza.

Una visión más de conjunto nos la proporciona la tabla que hemos elaborado, relativa a las Autonomías:

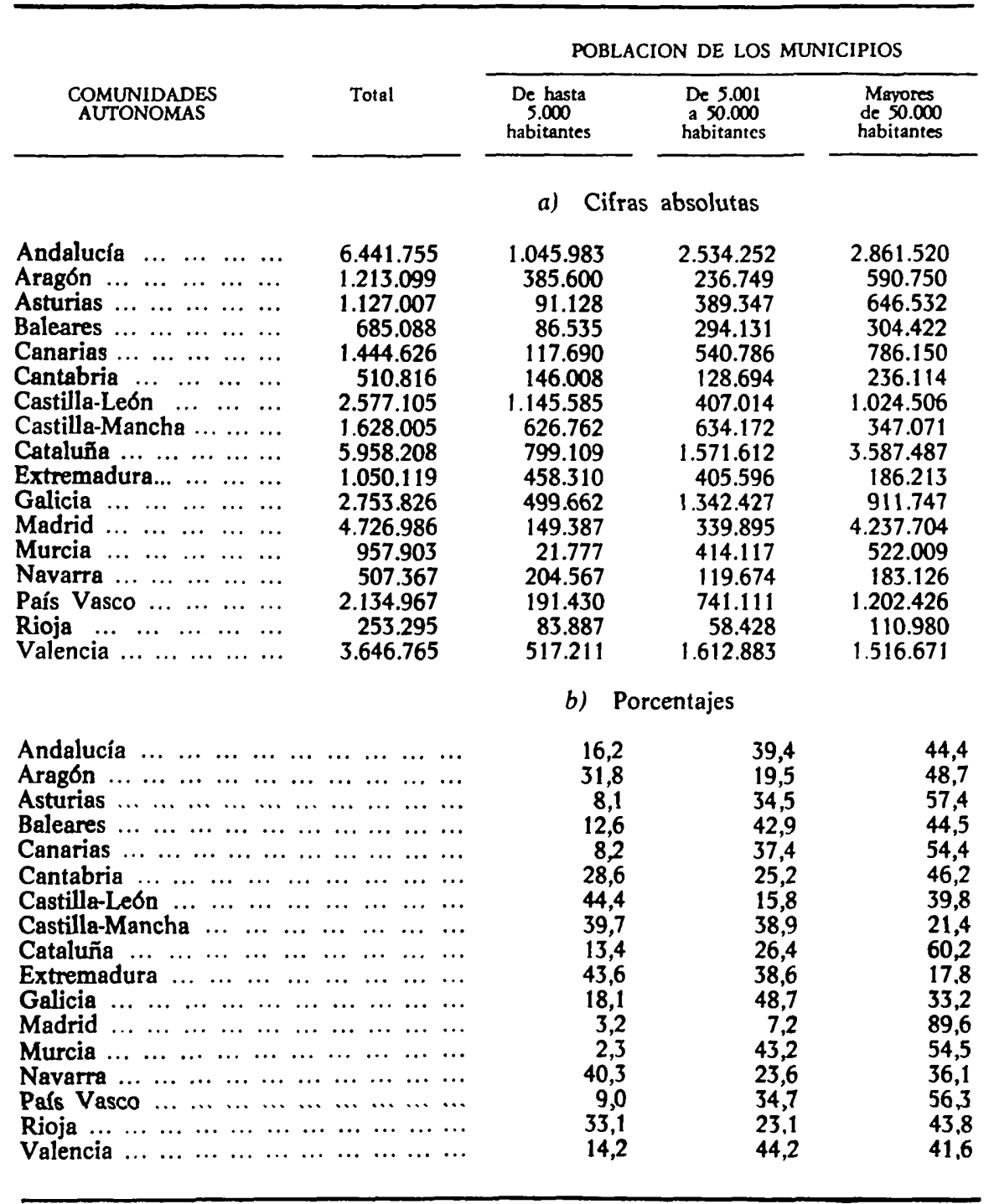


Los datos de las Comunidades Autónomas nos permiten perfilar la distribución de la población residente en los Municipios de hasta 5.000 habitantes, restringiendo su área, de esta forma:

- Noroeste y Norte.-Galicia y Cantabria;

- La Meseta._Ambas Castillas y Extremadura en su integridad, salvo la excepción de Madrid;

- Valle del Ebro.--Navarra y la Rioja,

de manera que no se confirma el carácter predominante ni en el litoral oriental mediterráneo ni en Andalucía.

Con respecto a la población residente en Municipios de tipo medio de entre 5.001 a 50.001 habitantes, existe una mayor identidad entre las Provincias y las Comunidades Autónomas, en orden a la distribución geográfica predominante, salvo algún matiz. Y así se configuran, con los datos de las Autonomías, estas zonas:

- Noroeste y Norte.-Galicia, Asturias y el País Vasco;

- Submeseta Sur.-Comprendiendo a Castilla-Mancha y a Extremadura;

- Litoral oriental mediterráneo.-Valencia, Murcia y Baleares;

- Andalucía;

- Canarias,

perdiendo personalidad, a estos efectos, la Submeseta Norte, que no aparece representada.

Finalmente, la población residente en Municipios superiores a los 50.000 habitantes destaca por su mayor peso en el conjunto provincial de Madrid, Cataluña; Asturias y el País Vasco; Murcia y Canarias.

Se perfilan también dos grandes áreas. La primera interior, que comprende las dos grandes Regiones naturales de la Meseta y el Valle del Ebro, pero ambas más debilitadas en relación con las conclusiones fijadas respecto al número de Municipios, ya que la primera se contempla en toda su unidad sólo con referencia a la población de los Municipios de hasta 5.000 habitantes, se reduce a la Submeseta Sur en cuanto a la población de los Municipios de tamaño medio y pierde su identidad con respecto a la de los grandes Municipios, manteniéndose sólo Madrid. El Valle del Ebro mantiene su personalidad solamente con respecto a los Municipios pequeños.

La segunda gran área, la periférica, se mantiene, aunque incom- 
pleta si la comparamos con la de los Municipios. Sólo se la contempla en su plenitud en cuanto a los Municipios de tamaño medio, puesto que con respecto a los Municipios pequeños no aparecen ni Andalucia ni Canarias.

La información disponible permite estudios más amplios y profundos contemplando el estudio de los grupos de Municipios considerados en el apartado II de este trabajo, tanto con respecto al número de Municipios como a la distribución de su población respectiva sobre el área territorial de España. 
REVL-1982, núm. 215. BALLESTER ROS, IGNACIO. LA POBLACION DE LOS MUNICIPIOS ESPAÑOLES.

REVL-1982, núm. 215. BALLESTER ROS, IGNACIO. LA POBLACION DE LOS MUNICIPIOS ESPAÑOLES. 


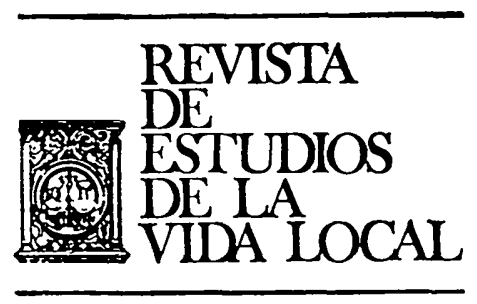

IV. JURISPRUDENCIA 
REVL-1982, núm. 215. BALLESTER ROS, IGNACIO. LA POBLACION DE LOS MUNICIPIOS ESPAÑOLES.

REVL-1982, núm. 215. BALLESTER ROS, IGNACIO. LA POBLACION DE LOS MUNICIPIOS ESPAÑOLES. 